\title{
Systemic effects of inhaled corticosteroids on growth and bone turnover in childhood asthma: a comparison of fluticasone with beclomethasone
}

\author{
R. Rao*, R.K. Gregson*, A.C. Jones*, E.A. Miles*, M.J. Campbell**, J.O. Warner*
}

Systemic effects of inhaled corticosteroids on growth and bone turnover in childhood asthma: a comparison of fluticasone with beclomethasone. R. Rao, R.K. Gregson, A.C. Jones, E.A. Miles, M.J. Campbell, J.O. Warner. (C) ERS Journals Ltd 1999.

ABSTRACT: Inhaled steroids are frequently used in childhood asthma, but concerns based on limited objective evidence remain, regarding long-term side-effects. In this study the systemic effects of standard doses of inhaled steroids in childhood asthma were assessed, comparing beclomethasone dipropionate (BDP) with fluticasone propionate (FP).

The study was prospective, randomized and double-blind. Twenty-three steroidnaive children with moderately severe asthma, aged 5-10 yrs, were allocated either BDP $\left(400 \mu \mathrm{g} \cdot \mathrm{day}^{-1}\right)$ or FP $\left(200 \mu \mathrm{g} \cdot \mathrm{day}^{-1}\right)$ using a metered-dose inhaler with a spacer. Asthma control was assessed at regular intervals over 20 months. Fasting morning blood and overnight urine samples were collected for estimation of serum cortisol, serum 1-carboxyterminal telopeptide (1CTP), serum osteocalcin and urine deoxypyridinoline (DPD). Bone mineral density (BMD) was measured at each visit.

None of the markers of bone turnover showed any change during the study period. BMD increased at normal rates with age. Serum cortisol significantly decreased on BDP, but not on FP. A significant difference in growth rates was found between the groups, with a slower rate of growth towards the end of the observation period in the BDP group.

In conclusion when taken in a relatively modest dose over a period of time, beclomethasone dipropionate had significant effects on the hypothalamic-pituitaryadrenal axis and statural growth in childhood asthma. These systemic effects were not seen with an equipotent dose of fluticasone propionate.

Eur Respir J 1999; 13: 87-94.

Corticosteroids are widely used as anti-inflammatory drugs in asthma. Oral steroids were introduced more than 40 yrs ago. There is clear evidence of systemic effects with oral steroids on adrenal function, growth and bone metabolism [1-5] when used to treat children with asthma. In addition, oral steroids have detrimental effects on carbohydrate, protein and fat metabolism, leading to cushingoid changes and hyperglycaemia. The introduction of inhaled steroids with high topical potency has avoided many of these side-effects, making them the mainstay of treatment [6]. There are now calls to use these in ever-increasing doses at earlier ages in milder disease. There are concerns, however, of systemic effects even with these drugs, especially in the growing child. There is some evidence of systemic effects with the use of inhaled steroids, in adults [7] as well as in children [8, 9]. However, the data so far available in children have shown discordant results, with some attributing delayed growth to the chronic disease rather than the treatment $[10,11]$.

There is some evidence of the effects of inhaled steroids on endogenous cortisol production. Beclomethasone dipropionate (BDP) has been shown to produce adrenal suppression in therapeutic doses [12-14]. It has been claimed that fluticasone propionate (FP), with its better safety profile, is much less likely to produce endogenous cortisol
Depts of $*$ Child Health and $* *$ Medical
Statistics and Computing, University of Southampton, Southampton, UK.

Correspondence: R. Rao

James Paget Hospital

Gorleston

Great Yarmouth

Norfolk NR31 6LA

UK

Fax: 441493453193

Keywords: Asthma

bone mineral density

1-carboxy-terminal telopeptide

cortisol

deoxypyridinoline

growth

Received: April 201997

Accepted after revision September 31998 suppression at equipotent doses [15-17]. The clinical relevance of these measures of systemic effects remains to be established. There is, therefore, an urgent need for further studies in childhood asthma comparing the original inhaled steroid, BDP, with newer alternatives, in conventional doses. These should ideally be medium- to long-term, initially excluding the confounding effects of puberty, but ultimately following through to adulthood.

This study was set up with the aim of looking at medium-term systemic effects of inhaled steroids over 20 months in a group of prepubertal children with moderately severe asthma, comparing BDP with the newer compound FP. The authors use $400 \mu \mathrm{g} \cdot \mathrm{day}^{-1}$ BDP as a starting dose in children of this age and, therefore, this was the dose chosen for the study. FP has been shown to be equipotent in half the dose of BDP [16], thus making a dose of 200 $\mu \mathrm{g} \cdot \mathrm{day}^{-1}$ appropriate for comparison.

\section{Subjects and methods}

\section{Subjects}

The study was designed to involve children with moderately severe asthma, whose control was unsatisfactory and who would have been starting prophylactic therapy as per international guidelines [6]. It was extremely difficult 
to find children in the required age group of 5-10 yrs who had not already been started on inhaled steroids. This is probably a reflection of the success of publicity promoting the early use of inhaled steroids.

Twenty-three steroid-naive children with moderately severe asthma were entered into the study after meeting the inclusion criteria during a two-week run-in period. Of these, two did not enter the extended phase of $1 \mathrm{yr}$ owing to late recruitment and constraints of time. The overall period of recruitment was $1 \mathrm{yr}$. No patients dropped out of the study. In order to achieve high levels of compliance, there were frequent clinic visits, reinforced by telephone contact between visits. Inhaler techniques and diary cards were checked carefully on every visit. Subjects were encouraged to contact the team at any time if they were having problems with asthma control. The age range of 5-10 yrs was selected for the following reasons. The lower age limit was set by the children's ability to perform reliable and repeatable spirometry, peak flows and treadmill exercise tests, while the upper age limit was set to exclude changes in physical and physiological parameters due to puberty and to take advantage of the almost linear growth rates during this period.

None of the patients had received oral steroids in the 2 weeks preceding entry into the study. Entry was delayed if there was evidence of a respiratory tract infection in the preceding 2 weeks. The subjects also had to demonstrate at least one of the following features: 1) asthma symptoms of at least a score of 1 (out of a maximum of 6, scores of 0-2 each for daytime, night-time and exercise-induced symptoms) on at least 2 separate days out of 7 consecutive days recorded in the diary card during the run-in period; 2) diurnal variation in peak flows $\geq 20 \%$ on at least 2 out of 7 consecutive run-in days; and 3) one acute exacerbation of asthma during the previous month.

Ethical approval was obtained from the Southampton joint ethics committee. Written informed consent was obtained from a parent and the child at the time of entry into the run-in period.

\section{Protocol}

The study was a randomized, double-blind, parallelgroup study, with placebo control for an initial period of 10 weeks (fig. 1). The children were randomized into three groups and started on metered-dose inhalers (MDI) with Volumatic $^{\mathrm{TM}}$ spacers (GlaxoWellcome, Middlesex, UK): group 1 received BDP $200 \mu \mathrm{g}$ twice daily, group 2 received FP $100 \mu \mathrm{g}$ twice daily and group 3 received placebo (only for the first 10 weeks) 2 puffs twice daily.

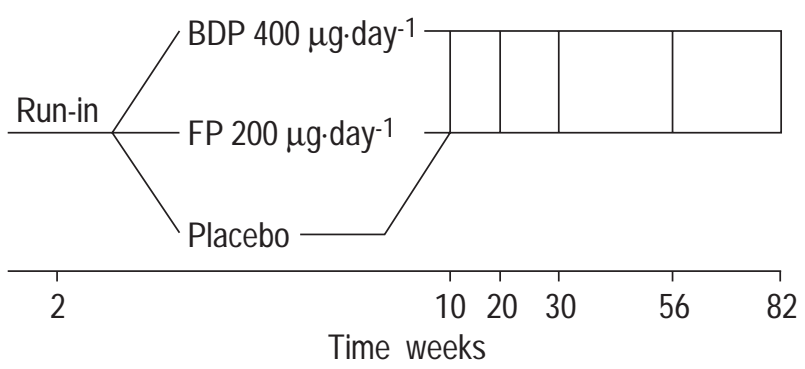

Fig. 1. - Study protocol. BDP: beclomethasone dipropionate; FP: fluticasone propionate.
Although a control group for the entire 20-month period would have been ideal, it was considered ethically unacceptable to continue children with significant symptoms on placebo for such a length of time. At 10 weeks into the study, the placebo group was merged with group 2 . This was carried out as there was a paucity of long-term data on FP and resulted in a 2:1 distribution of subjects on FP versus BDP (15 versus 8 ). The subjects were seen at 2 weeks for a blood and urine test. They had regular clinic visits at 10 -weekly intervals for the first 30 weeks, then at 6 monthly intervals for an extended phase of a further year. Telephone contact was made halfway between clinic visits. The total duration of the study was 20 months. Concomitant use of sodium cromoglycate, started prior to recruitment, was allowed (two children). None of the patients were using long-acting $\beta_{2}$-agonists or theophyllines.

\section{Clinical measurements}

At each clinic visit, asthma control was assessed using diary cards, spirometry, exercise and histamine challenges. Diary cards contained day and night symptom scores, twice-daily peak flow measurements using a Mini-Wright peak flow meter and frequency of rescue medication use. Scores were out of a maximum of 6 . Acute asthma exacerbations were also noted, as were any other illnesses. Other than an occasional day when the child or parent forgot to fill in the card, or when the child was away from home and peak flows could not be performed, the cards were mostly complete. Diary card data were collected for only the initial 8 months, as for the extension period of the study it was felt inappropriate to ask the families to fill in daily records for a whole year, with a clinic visit made only at 6-month intervals. This could have led to incomplete and retrospective recording, with potential recall bias.

\section{Laboratory measurements}

Fasting 09:00 h blood and overnight urine samples were collected at each visit for the estimation of serum cortisol, serum 1-carboxyterminal telopeptide (1CTP), serum osteocalcin and urine deoxypyridinolines (DPD). Serum osteocalcin was measured as a marker of osteoblast activity (bone formation), while serum 1CTP and urine DPD were assayed as markers of osteoclast activity (bone resorption). Blood samples were centrifuged and serum stored at $-70^{\circ} \mathrm{C}$ until analysis. Urine was aliquoted into 5-mL samples and stored at $-70^{\circ} \mathrm{C}$.

The recently developed monoclonal immunoassay from Pharmacia Diagnostics (Uppsala, Sweden) was used for measuring serum osteocalcin. The advantage of this assay is that it only measures the intact osteocalcin molecule, the monoclonal antibody (mAb 689), depending on the conformity of the native molecule. It thus avoids errors due to detection of fragments created during resorption [18]. This is claimed to enhance its specificity significantly compared with previous assays. It is essentially a fluorescent immunoassay using the Pharmacia ImmunoCAP ${ }^{\mathrm{TM}}$ and $\mathrm{CAP}^{\mathrm{TM}}$ system. Reference ranges for children under 9 yrs were: mean $25 \mu \mathrm{g} \cdot \mathrm{L}^{-1}$, range $(95 \%$ confidence interval (CI)) $18-35 \mu \mathrm{g} \cdot \mathrm{L}^{-1}$, detection limit $<0.5 \mu \mathrm{g} \cdot \mathrm{L}^{-1}$, interassay coefficients $<8 \%$ and intra-assay coefficients $<7.2 \%$. 
Serum 1CTP was measured using a radioimmunoassay (RIA) kit (Orion Diagnostica, Espoo, Finland); $100 \mu \mathrm{L}$ serum sample was mixed with $200 \mu \mathrm{L}{ }^{125}$ I-labelled 1CTP. The two antigens were allowed to compete for the limited number of binding sites on $200 \mu \mathrm{L} 1 \mathrm{CTP}$ antiserum. After washing away the excess antigen, the radioactivity in the precipitated antigen-antibody complex was counted on a gamma counter and actual concentration of 1CTP calculated from a standard curve, on the principle that radioactive antigen in the antigen-antibody complex is inversely proportional to the sample antigen. Reference ranges for children aged 4-10 yrs were: mean $11 \mu \mathrm{g} \cdot \mathrm{L}^{-1}$, range $(95 \%$ CI) $8-18 \mu \mathrm{g} \cdot \mathrm{L}^{-1}$, detection limit $0.5 \mu \mathrm{g} \cdot \mathrm{L}^{-1}$, interassay coefficients $<6.7 \%$ and intra-assay coefficients $<3.3 \%$.

Urine DPD was measured using Pyrilinks-D ${ }^{\mathrm{TM}}$ (Metro Biosystems, UK). This recently developed rapid assay shows excellent correlations with measurements made using the accurate but tedious ion-paired reversed-phase highprecision liquid chromatography. It works as a competitive enzyme immunoassay in a microtitre-plate format, utilizing a monoclonal antibody against DPD coated on the plate. Free DPD in the urine sample competes against a conjugate of DPD-alkaline phosphatase for the antibody. The reaction is detected using a substrate, the optical density being read at $405 \mathrm{~nm}$. A four-parameter curve fit is needed for accurate results. The results were corrected for urine creatinine and expressed as nM DPD.mmol of creatinine ${ }^{-1}$. Reference ranges for children aged 5-10 yrs were: mean $26 \mathrm{nM} \cdot \mathrm{mmol}$ creatinine $^{-1}$, range $11-49 \mathrm{nM} \cdot \mathrm{mmol}$ creatinine $^{-1}$, detection limit $1.1 \mathrm{nM} \cdot \mathrm{mmol}$ creatinine ${ }^{-1}$, intra-assay coefficients $<9.5 \%$ and interassay coefficients $<10.3 \%$.

Fasting serum cortisol levels were measured at 09:00 h on each visit for every child, to assess systemic effects of the inhaled steroids on the hypothalamic-pituitary-adrenal (HPA) axis. The blood samples were assayed at the clinical trials unit at West Middlesex Hospital. The method employed was an enzyme linked immunoassay (EnzymunTest System ES 300 TM; Boehringer Diagnostics, UK). The normal value range for children was $100-500 \mathrm{nmol} \cdot \mathrm{L}^{-1}$. The minimum detection limit was $27.6 \mathrm{nmol} \cdot \mathrm{L}^{-1}$. Interassay coefficients of variation were $<4.2 \%$ and intra-assay coefficients were $<4.3 \%$.

Bone mineral density (BMD) measurements of lumbar spine and total body were carried out by dual-energy Xray absorptiometry (DEXA), using a Lunar ${ }^{\mathrm{TM}}$ densitometer (Lunar, USA). Bone density is a measure of architecture, a three-dimensional structural reflection of changes in bone turnover over a prolonged period. The spine is mainly formed of trabecular bone, turnover of which is much more rapid than that of cortical bone, especially in the growing child. This makes it a sensitive region in which to measure subtle changes, as would occur with the use of steroids.

At the same time during each visit (10:00-10:30 h), the children had their height measured using a Harpenden stadiometer. The mean value of three measurements was recorded. All height measurements were carried out by one of two observers (R. Gregson or E. Miles) using the same technique, with the child steadied by slight upward pressure on the angle of mandible and the head in the "Frankfurt plane" (external auditory meatus lined up horizontally with lower margin of orbit). Height measurements were converted into standard deviation scores (SDS) using the new British reference data [19]. All of the children were rated as being prepubertal throughout the study.

\section{Statistical analysis}

All data were entered into an SPSS $^{\mathrm{TM}}$ database (SPSS Inc., Chicago, IL, USA). For statistical analyses, data were split into three subgroups (including the placebo group) for the first 10 weeks and two subgroups (BDP and FP) thereafter. Regression analyses were performed on BMD measurements and markers of bone turnover. Peak flow variability, a measure of asthma control, was assessed using standard deviations of average monthly peak flow for each subject. Regression coefficients were then calculated over time to look for changes in standard deviations in peak flows as evidence of asthma control. Slopes of the two groups on BDP and FP were compared. Similarly, monthly means of daily symptom scores were used for each subject to calculate regression coefficients against time and regression slopes of the two treatment groups were compared. Parameters of airway calibre (forced expiratory volume in one second (FEV1) and forced midexpiratory flow (FEF25-75\%)), exercise- and histamineinduced bronchial hyperresponsiveness were plotted and regression analyses performed. Further detailed statistical analyses were performed using the STATA 5 statistical package for cross-sectional time-series longitudinal analysis with a random effects term to allow for betweensubject variation (Stata Corp., USA).

\section{Results}

\section{Clinical efficacy}

The baseline data for all the subgroups are given in table 1. The groups were well matched, except for serum immunoglobulin (Ig)E levels, which were significantly higher in the BDP group. Parameters of airway calibre, FEV1, and FEF25-75\%, showed an improvement in the first 20 weeks in the whole group (paired sample t-test for FEV1, $\mathrm{p}=0.018$ ), this was maintained thereafter but no further improvements were seen (fig. 2). Similarly, the exercise-induced drop in FEV1, and histamine-induced bronchoconstriction (provocative concentration of histamine causing a 20\% fall in FEV1 (PC20)) showed a response in the initial few weeks. Linear regression against time showed only the BDP group attaining significance (table 2). Daily symptom scores showed a significant reduction for the whole study group (regression slope $(B)$ : $-0.0571 ; \mathrm{p}<$ $0.0001)$. There was no detectable difference between the two drug groups (fig. 3). Peak flow standard deviation scores reduced significantly over the same period $(B:-0.7066$; $\mathrm{p}=0.003$ ). However, the BDP group started with a higher variability, the reason for which is unclear. The slopes for the two groups did not differ (fig. 4). Thus, BDP (400 $\mu \mathrm{g}$. day $\left.^{-1}\right)$ and FP $\left(200 \mu \mathrm{g} \cdot\right.$ day $\left.^{-1}\right)$ did not show any detectable difference in clinical efficacy.

\section{Markers of bone turnover}

None of the markers of bone turnover showed any significant change with inhaled corticosteroid treatment, either for the group as a whole (table 3) or for subgroups based on drug therapy. Significant correlations were found 
Table 1. - Baseline data for the whole group and by treatment subgroups

\begin{tabular}{lcccc}
\hline Variable & Total & BDP & FP & Placebo \\
\hline Age yrs & $6.77 \pm 0.61$ & $6.93 \pm 0.61$ & $6.68 \pm 0.57$ & $6.69 \pm 0.61$ \\
Height cm & $120.0 \pm 2.0$ & $121.8 \pm 3.4$ & $117.3 \pm 2.9$ & $121.2 \pm 4.4$ \\
Weight kg & $23.26 \pm 1.12$ & $22.47 \pm 1.51$ & $22.82 \pm 1.26$ & $24.68 \pm 3.10$ \\
Cortisol nmol.L -1 & $279.6 \pm 27.3$ & $334.2 \pm 34.3$ & $282.0 \pm 53.9$ & $215.0 \pm 49.9$ \\
IgE IU·L & $506.3 \pm 109.2$ & $857.5 \pm 243.4$ & $228.8 \pm 76.4$ & $421.7 \pm 132.1$ \\
FEV1 \% pred & $87.9 \pm 3.1$ & $79.3 \pm 5.5$ & $90.8 \pm 4.7$ & $94.4 \pm 4.7$ \\
FEF25-75\% \% pred & $78.1 \pm 6.1$ & $68.3 \pm 10.2$ & $90.1 \pm 7.9$ & $86.0 \pm 10.1$ \\
Exercise \% drop in FEV1 & $21.6 \pm 3.4$ & $30.4 \pm 4.2$ & $18.6 \pm 6.4$ & $21.0 \pm 6.0$ \\
PC20 for histamine & $2.07 \pm 0.5$ & $0.32 \pm 0.09$ & $2.68 \pm 0.66$ & $2.65 \pm 1.17$ \\
PEFR \% pred & $84.9 \pm 3.1$ & $81.3 \pm 5.7$ & $86.5 \pm 4.4$ & $87.1 \pm 6.8$ \\
\hline
\end{tabular}

Data are shown as mean \pm SEM. BDP: beclomethasone dipropionate; FP: fluticasone propionate; IgE: immunoglobulin E; FEV1: forced expiratory volume in one second; FEF25-75\%: forced mid-expiratory flow; PC20: provocative concentration of histamine causing a $20 \%$ fall in FEV1; PEFR: peak expiratory flow rate.

between the regression coefficients of 1CTP and osteocal$\operatorname{cin}(r=0.47, p=0.02)$ and between 1CTP and DPD in both subgroups $(\mathrm{r}=0.76$ and $0.71, \mathrm{p}=0.02$ and 0.05 , respectively). BMD followed the normal pattern of increase with age for both young females and males. No correlation was found, either at baseline or over time, between BMD measurements and markers of bone turnover.

\section{Hypothalamic-pituitary-adrenal axis}

When serum cortisol was analysed, in the BDP group there was a trend towards a decrease in levels by 2 weeks into treatment. By 10 weeks there was a significant drop in mean cortisol level, although not below normal limits $\left(100-500 \mathrm{nmol} \cdot \mathrm{L}^{-1}\right)$, which persisted throughout the 20 month period. No changes were observed in cortisol levels in the FP group (table 4).

\section{Height}

A highly significant difference was observed as regards long-term linear growth in the two groups. The FP group grew on average by $5.75 \mathrm{~cm} \cdot \mathrm{yr}^{-1}$. This would be on the 50 th centile of accepted prepubertal growth rates in heal-

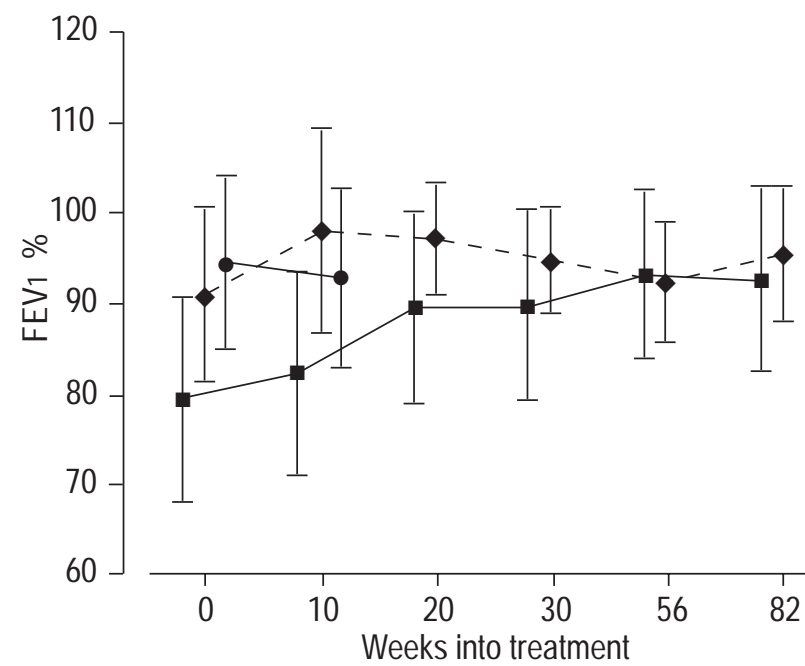

Fig. 2. - Changes in forced expiratory volume in one second (FEV1) over time by treatment subgroups (mean \pm SEM). $\mathbf{a}$ : beclomethasone; fluticasone; $\bullet$ : placebo. thy individuals of both sexes. There was no difference between the FP group and placebo group in growth rates in the first 10 weeks. The BDP group, however, grew significantly more slowly, at an average rate of $4.94 \mathrm{~cm} \cdot \mathrm{yr}^{-1}$ ( 0.4 th centile on growth charts), a difference of 0.81 $\mathrm{cm} \cdot \mathrm{yr}^{-1}$ (95\% CI: $\left.0.45-1.16\right)$. This difference in growth rates persisted throughout the study. To eliminate the confounding effect of differential growth rates at different centiles, height standard deviation scores (SDS; z-scores) were estimated. Comparison of $\mathrm{z}$ scores supported the earlier findings, with a significant difference in regression coefficients between the two groups (fig. 5). What was striking was that the BDP group almost universally showed a decrease in height SDS during the latter period of the study, a trend notably absent in the FP group (fig. 6).

\section{Discussion}

While this study involved small numbers, the analysis of symptom scores and peak flows employed a very large number of data points, thus allowing for significance to be assessed with some accuracy. The objective parameters of airway calibre and function showed a tendency to improve over the first few weeks and then stabilize, although these reached significance only in the BDP group. Means of

Table 2. - Linear regression coefficients for lung function parameters over time

\begin{tabular}{llccc}
\hline Variable & Treatment & $\begin{array}{c}\text { Regression } \\
\text { coefficient }(B)\end{array}$ & $\begin{array}{c}\text { SE } \\
\text { for } B\end{array}$ & p-value \\
\hline FEV1 \% & BDP & 0.470 & 0.118 & 0.0002 \\
& FP & 0.032 & 0.063 & 0.606 \\
& Placebo & 1.198 & 0.446 & 0.019 \\
FEF25-75\% & BDP & 1.120 & 0.166 & $<0.0001$ \\
& FP & 0.126 & 0.122 & 0.303 \\
& Placebo & 2.146 & 0.840 & 0.025 \\
\% drop in FEV1 & BDP & -0.399 & 0.144 & 0.008 \\
with exercise & FP & -0.044 & 0.044 & 0.327 \\
& Placebo & -1.734 & 0.470 & 0.003 \\
Log PC20 for & BDP & 0.018 & 0.003 & $<0.0001$ \\
histamine & FP & -0.003 & 0.002 & 0.292 \\
& Placebo & 0.065 & 0.027 & 0.036 \\
\hline
\end{tabular}

FEV1: forced expiratory volume in one second; FEF25-75\%: forced mid-expiratory flow; $\mathrm{PC} 20$ : provocative concentration of histamine causing a $20 \%$ fall in FEV1; BDP: beclomethasone dipropionate; FP: fluticasone propionate. 


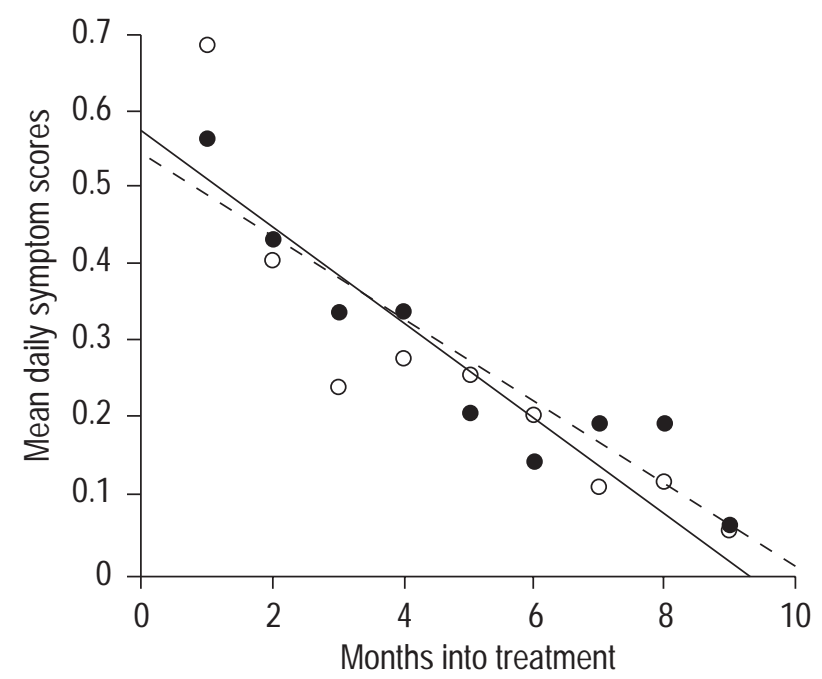

Fig. 3. - Multiple regression analysis of mean symptom scores over time. $B$ (slope for beclomethasone; $\bigcirc$ ): -0.057 , SE of $B=0.005, \mathrm{p}<$ 0.0001 ; difference for fluticasone $(\bullet)$ : $\mathrm{p}=0.889$.

daily symptom scores improved very significantly over the initial 8-month period, with no difference between BDP and FP. Peak flow variability similarly demonstrated a significant narrowing in the group as a whole. The observed difference in starting points between the two groups was a chance finding and was associated with higher IgE levels in the BDP group, which probably led to a higher degree of airway inflammation, compared with the FP group (table 1). Crucially, however, the two slopes ran in parallel, indicating that the clinical improvement was similar in the two groups. This finding assumes added significance when related to the difference in side-effect profiles of the two drugs on growth and cortisol suppression.

Although spot serum cortisols are not the most sensitive way to assess the effects of inhaled steroids on the HPA axis, the fact that significant changes in endogenous cortisol production were demonstrated with BDP would suggest a possible systemic effect. A similar effect on spot cortisol was shown by Doull et al. [20]. Phillip et al. [14]

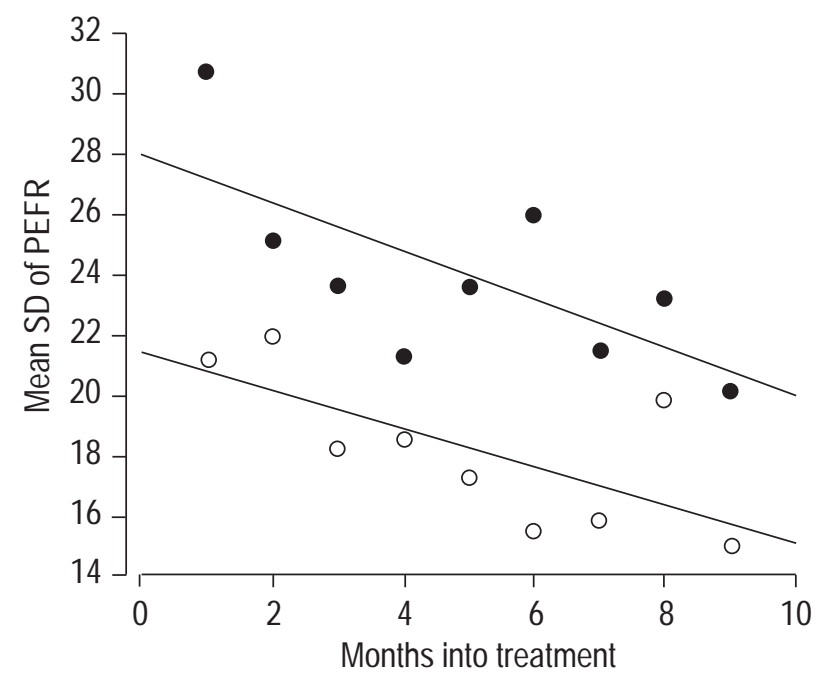

Fig. 4. - Multiple regression analysis of peak flow variability (SD of PEFR) over time. $B$ (slope for beclomethasone; $\bigcirc$ ): -0.706 , SE of $B=$ $0.203, \mathrm{p}<0.003$; difference for fluticasone $(\bullet)$ : $\mathrm{p}=0.001$.
Table 3. - Means of regression coefficients for markers of bone turnover over 20 months

\begin{tabular}{lrc}
\hline Variable & \multicolumn{1}{c}{ Mean } & $95 \%$ CI for mean \\
\hline Osteocalcin & 0.0232 & $-0.0236-0.0699$ \\
Serum 1CTP & -0.0225 & $-0.0418--0.0031$ \\
Urine DPD & -0.0090 & $-0.0209-0.0021$ \\
BMD (LS) & 0.0005 & $0.0002-0.0007$ \\
BMD (TB) & 0.0004 & $0.0003-0.0005$ \\
\hline
\end{tabular}

CI: confidence interval; 1CTP: 1-carboxy-terminal telopeptide; DPD: deoxypyridinoline; BMD: bone mineral density; LS: lumbar spine; TB: total body.

in 1992, using repeated measurements of plasma cortisol, showed similar results to the present study in children on long-term BDP. Studies in children, where plasma cortisol levels were measured at frequent intervals over a 24-h period, have shown that $400-1,000 \mu \mathrm{g}$ of BDP.day ${ }^{-1}$ produced a significant reduction in the physiological secretion of cortisol [12, 21]. WYATT et al. [22] in 1978 and CHANG and TAM [23] in 1991 have also shown significant changes in urinary cortisol excretion in children on 400 $\mu \mathrm{g} \cdot \mathrm{day}^{-1} \mathrm{BDP}$ as dry powder as well as MDI, although the levels stayed within the normal range. Sustained, but not cumulative, effects on the HPA axis by inhaled steroids have also been demonstrated in a study by NIKOLAIZIK et al. [24] over a 1-yr period. These results have to be balanced against the conclusions of other studies which did not find significant variation in either urine or plasma cortisol levels at various doses of BDP $[25,26]$. In the present study, a dose of $200 \mu \mathrm{g} \cdot \mathrm{day}^{-1}$ of FP did not show any detectable change in serum cortisol. This would confirm the findings of earlier studies on the reduced systemic bioavailability of FP in therapeutic doses and, thus, a diminished potential for systemic side-effects on prolonged use [15-17]. Using $24 \mathrm{~h}$ urinary excretion rates for cortisol

Table 4. - Changes in means of serum cortisol levels over time with beclomethasone dipropionate (BDP), fluticasone propionate (FP) and placebo (paired-sample t-tests)

\begin{tabular}{cccc}
\hline Weeks & Mean \pm SEM & p-value & $\begin{array}{c}95 \% \text { CI for } \\
\text { difference in means }\end{array}$ \\
\hline BDP group & & & \\
0 & $323.85 \pm 37.78$ & - & - \\
2 & $232.14 \pm 26.46$ & 0.070 & $-13.63-197.06$ \\
10 & $184.74 \pm 18.79$ & 0.010 & $44.64-254.50$ \\
20 & $201.78 \pm 20.74$ & 0.011 & $40.45-207.83$ \\
30 & $205.00 \pm 23.69$ & 0.003 & $62.47-196.02$ \\
56 & $208.25 \pm 17.85$ & 0.007 & $47.17-204.82$ \\
86 & $190.85 \pm 30.47$ & 0.013 & $35.77-228.80$ \\
FP group & & & - \\
0 & $281.50 \pm 59.93$ & - & $-66.79-175.45$ \\
2 & $246.33 \pm 21.36$ & 0.93 & $-149.91-260.25$ \\
10 & $236.33 \pm 34.86$ & 0.52 & $-58.32-128.89$ \\
20 & $213.21 \pm 24.86$ & 0.43 & $-63.23-81.38$ \\
30 & $239.42 \pm 24.94$ & 0.79 & $-115.70-71.70$ \\
56 & $251.83 \pm 39.13$ & 0.61 & $-99.90-141.54$ \\
86 & $214.45 \pm 29.72$ & 0.71 & - \\
Placebo group & & & $-69.70-86.84$ \\
0 & $215.00 \pm 49.97$ & - & \\
2 & $267.85 \pm 37.07$ & 0.32 & $-173.19-67.48$ \\
10 & $206.42 \pm 28.75$ & 0.79 & -6
\end{tabular}

CI: confidence interval. 


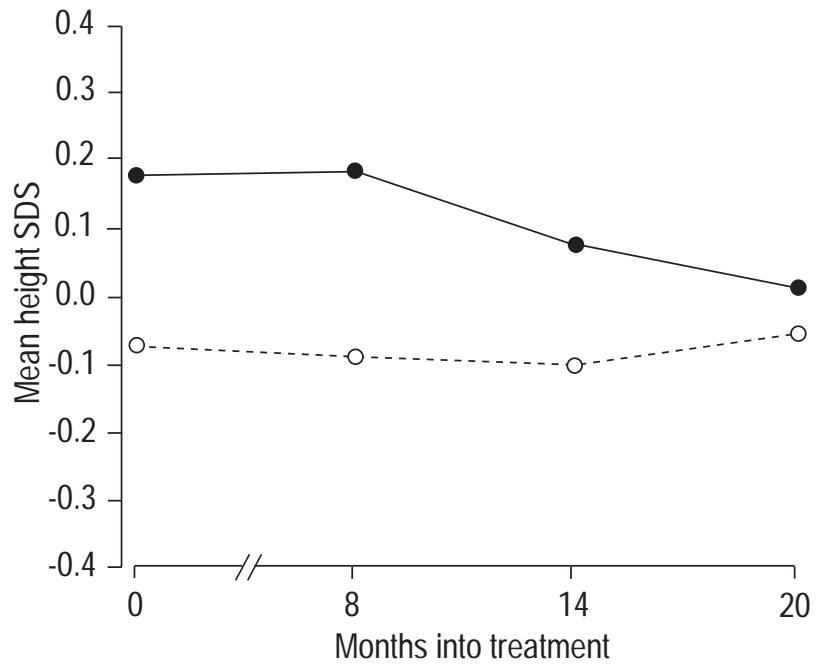

Fig. 5. - Time series regression analysis of height standard deviation scores (SDS) (z-scores). $\bullet$ : beclomethasone (BDP); $\bigcirc$ : fluticasone (FP). Slope for BDP: -0.137 ; slope for FP: 0.07; difference in slopes: 0.116; $\mathrm{p}<0.001 ; 95 \%$ confidence interval: $0.061-0.169$.

and its metabolites, Yiallouros et al. [27] have also recently demonstrated adrenal suppression with BDP but not with $\mathrm{FP}$, in equipotent doses.

Concerns remain regarding the effects of long-term use of inhaled steroids on bone and growth in the child. Studies from asthmatics have shown systemic effects with inhaled steroids [28] sometimes even in moderate doses [29]. However, the extent to which these affect bone is yet to be established. Most studies have either been cross-sectional or have followed their patients over short periods. It is well known that children with poorly controlled moderate-to-severe asthma tend to have decreased physical activity which, in itself, could lead to osteoporotic changes. It is essential that such confounding factors are taken into consideration and that the study group be as representative as possible. Serum osteocalcin has been the most widely studied of the biochemical markers of bone turnover. Studies using oral and high-dose inhaled steroids in asthma have shown reductions in serum osteocalcin levels $[2,8$, 9], whereas with inhaled steroids, the results have been inconsistent $[30,31]$. The effect on bone mass with the usage of inhaled steroids was not found to be significant in a recent cross-sectional study [32]. Significant correlates between various markers of bone turnover in the present study confirm that bone turnover is a dynamic process in which bone formation and resorption are taking place in closely coupled equilibrium. In the doses used, neither BDP nor FP altered this equilibrium. Lack of any correlation either at baseline or over time, between BMD measurements and markers of bone turnover, would suggest that these reflect different aspects of bone metabolism, the markers measuring the dynamics of continuous remodelling, whereas densitometry reflects long-term structural change. The results led us to postulate that, when used in standard doses over a prolonged period in children with moderately severe asthma, neither BDP nor FP has a deleterious effect on either bone turnover or bone architecture. However, there may be a differential effect dependent on age, with adults having a greater risk of adverse effects on bone.
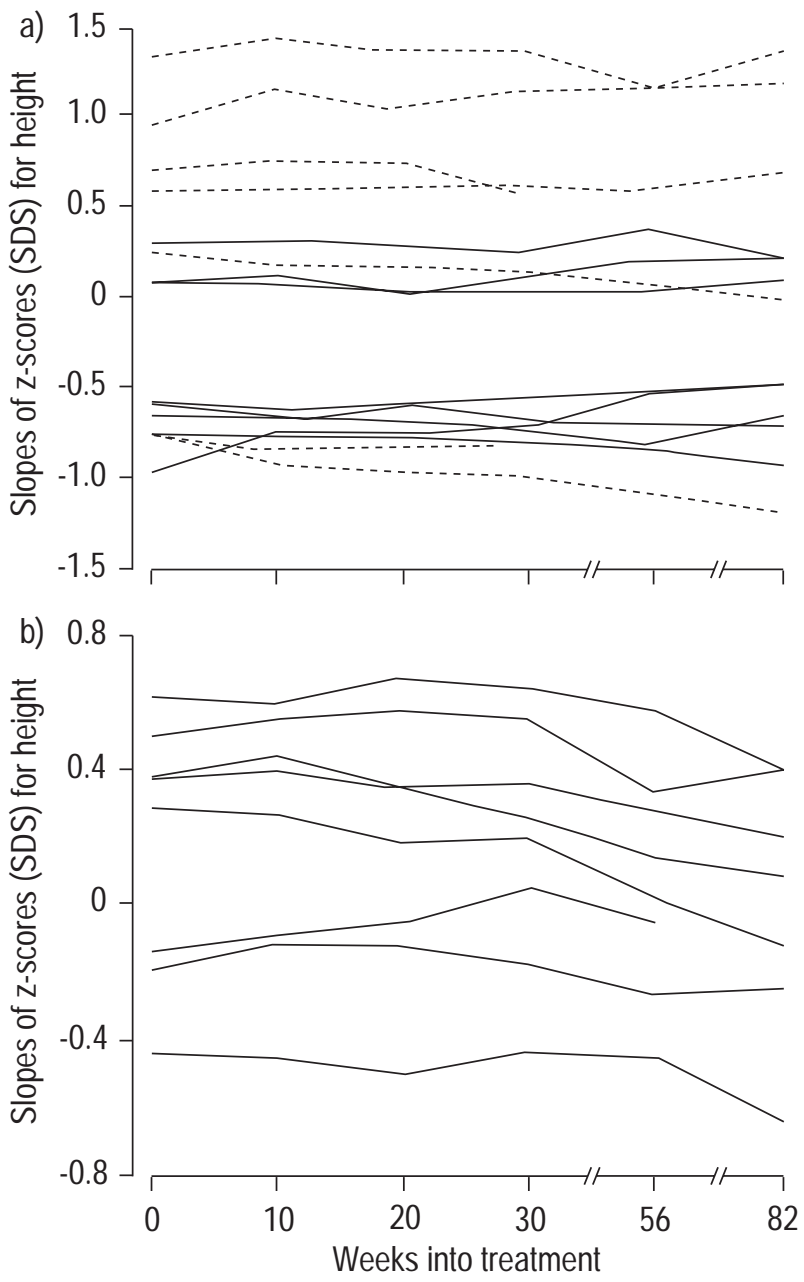

Fig. 6. - Individual slopes of height standard deviation scores (SDS) (z-scores) by treatment group. a) Fluticasone (FP) group ( FP throughout; - . - . - : placebo/FP); b) beclomethasone group.

LiTTLEWOOD et al. [33] looked at growth rates in children treated with BDP compared with other forms of treatment. Although open to criticism owing to a mismatch in age between the groups, this study showed a decreased rate of growth in the BDP group. TinKLEMAN et al. [34] in 1993 described medium-term growth over a period of $1 \mathrm{yr}$ in a large group of children who were treated with either BDP or theophylline. Pubertal growth differences were not accounted for and could have influenced the results, but the study showed a significant decrease in mean growth in height in the BDP group, which was more pronounced in males. Recent studies have used knemometry (measurement of lower leg length) as a tool to assess growth. Wolthers and PeDERson [35] have published a series of papers using knemometry to assess the effects of prednisolone as well as BDP used in an open manner. These studies have shown a potential for corticosteroids at moderate doses to impair growth in the short-term. This does not necessarily translate into decreased long-term growth. The study by Doull et al. [20] over a longer period also showed a significant reduction in growth in children on BDP compared with those on placebo. Furthermore, they found no catch-up growth during a 5month washout period. Criticism of this study relates to the 
population studied, who would not normally be judged to need inhaled steroid prophylaxis as they had very mild asthma, making it inappropriate to extrapolate these results to moderate- to severe-asthmatic children.

The present study subjects were all prepubertal, thus avoiding the potential pitfall of differential pubertal growth spurts. A definite long-term effect on growth with BDP was demonstrated, even at a dose of $400 \mu \mathrm{g} \cdot$ day $^{-1}$. This effect was not seen with an equipotent dose of FP (200 $\left.\mu \mathrm{g} \cdot \mathrm{day}^{-1}\right)$. The results were supported by similar differences in height SDS, showing a tailing off in growth during the last 12 months of the study. These findings confirm that the detrimental effect seen in studies into short-term growth with BDP $[20,34,35]$ persists long term in children even when given appropriate doses of the drug. CROWLEY et al. [36] compared growth rates over $1 \mathrm{yr}$ between groups of asthmatic children. They found that all of the steroid formulations affected growth, with oral prednisolone having the most suppressive effect. However, these children were on varying doses and using different inhalant systems. A recent publication by VERBERNE et al. showed a significant slowing of growth in children on BDP in a dose of $400 \mu \mathrm{g} \cdot$ day $^{-1}$ by a powdered device over a period of $1 \mathrm{yr}$ [37]. Another recent study involving 241 children from Canada has also shown a decrease in linear growth over a period of $1 \mathrm{yr}$ in a subgroup on $400 \mu \mathrm{g} \cdot \mathrm{day}^{-1}$ BDP by powdered device [38]. However, in a large retrospective study, SiLVERSTEIN et al. [39] looked at attained adult heights in those treated with glucocorticoids for asthma and compared them with matched controls of asthmatics not treated with glucocorticoids. They did not find any difference in adult heights. These results are encouraging, but it is essential that prospective studies following children through puberty to adulthood are conducted to prove or refute these conclusions.

During periods of slow growth, interventions such as inhaled steroids may suppress the effects of small amounts of growth-stimulating factors, but during rapid growth spurts many other stimulants are present, which overcome the small effects of the inhaled corticosteroids. Thus, the effect on final height may well be negligible. Although no information exists to question the safety of the established inhaled steroid beclomethasone dipropionate in low doses, if higher doses are required to achieve good control in childhood asthma, serious consideration should be given to switching to an alternative with a better systemic effect profile.

\section{References}

1. Allen DB, Mullen M, Mullen B. A meta-analysis of the effect of oral and inhaled corticosteroids on growth. $J$ Allergy Clin Immunol 1994; 93: 967-976.

2. Kerrebijn KF, De Kroon JPM. Effect on height of corticosteroid therapy in asthmatic children. Arch Dis Child 1968; 43: 556-561.

3. Dempster DW. Bone histomorphometry in glucocorticoid-induced osteoporosis. J Bone Miner Res 1989; 4: 137-141.

4. Adinoff AD, Hollister JR. Steroid induced fractures and bone loss in patients with asthma. N Engl J Med 1983; 309: 265-268.

5. Meeran K, Hattersley A, Burrin J, Shiner R, Ibbertson K. Oral and inhaled corticosteroids reduce bone formation as shown by plasma osteocalcin levels. Am J Respir Crit Care Med 1995; 151: 333-336.

6. The British guidelines on asthma management - 1995 review and position statement. Thorax 1997; 52: S1-S20.

7. Barnes NC. Safety of high-dose inhaled corticosteroids. Respir Med 1993; 87: Suppl. A, 27-31.

8. Ali NJ, Capewell S, Ward MJ. Bone turnover during high dose inhaled corticosteroid treatment. Thorax 1991; 46: 160-164.

9. Pouw EM, Prummel MF, Oosting H, Roos CM, Endert E. Beclomethasone inhalation decreases serum osteocalcin concentrations. BMJ 1991; 302: 627-628.

10. Graff-Lonnevig V, Kreapelien S. Long term treatment with beclomethasone dipropionate aerosol in asthmatic children with special reference to growth. Allergy 1979; 34: $57-61$.

11. Ninan TK, Russell G. Asthma, inhaled corticosteroid treatment, and growth. Arch Dis Child 1992; 67: 703-705.

12. Law CM, Marchant JL, Honour JW. Nocturnal adrenal suppression in asthmatic children taking inhaled beclomethasone dipropionate. Lancet 1986; 26: 942-944.

13. Bisgaard H, Nielsen MD, Andersen B, et al. Adrenal function in children with bronchial asthma treated with beclomethasone dipropionate or budesonide. J Allergy Clin Immunol 1988; 81: 1088-1095.

14. Phillip M, Aviram M, Leiberman E, et al. Integrated plasma cortisol concentration in children with asthma receiving long-term inhaled corticosteroids. Pediatr Pulmonol 1992; 12: 84-89.

15. Harding SM. The human pharmacology of fluticasone propionate. Respir Med 1990; 84: Suppl. A, 25-29.

16. Gustafsson P, Tsanakas J, Gold M, Primhak R, Radford $\mathrm{M}$, Gillies E. Comparison of the efficacy and safety of inhaled fluticasone propionate $200 \mu \mathrm{g} /$ day with inhaled beclomethasone dipropionate $400 \mu \mathrm{g} /$ day in mild and moderate asthma. Arch Dis Child 1993; 69: 206-211.

17. Fabbri L, Burge PS, Croonenborgh L. On behalf of an international study group. Comparison of fluticasone propionate with beclomethasone dipropionate in moderate to severe asthma treated for one year. Thorax 1993; 48: 817823.

18. Carlsson L, Nyberg B-I, Thunberg R, Ryden A-C, Enander I. Serum osteocalcin-A clinical useful biochemical marker for monitoring the side effects of glucocorticoid treatment. Am J Respir Crit Care Med 1995; 151: A374.

19. Freeman JV, Cole TJ, Chinn S, Jones PRM, White EM, Preece MA. Cross sectional stature and weight reference curves for the UK, 1990.

20. Doull IJM, Freezer NJ, Holgate ST. Growth of prepubertal children with mild asthma treated with inhaled beclomethasone propionate. Am J Respir Crit Care Med 1995; 151: 1715-1719.

21. Tabacknik E, Zadik Z. Diurnal cortisol secretion during therapy with inhaled beclomethasone dipropionate aerosol in children with asthma. J Pediatr 1991; 118: 294 297.

22. Wyatt R, Waschek J, Weinberger M. Effects of inhaled beclomethasone dipropionate and alternate-day prednisolone on pituitary-adrenal function in children with chronic asthma. N Engl J Med 1978; 299: 1387-1391.

23. Chang CC, Tam AY. Suppression of adrenal function in children on inhaled steroids. J Paediatr Child Health 1991; 27: 232-234.

24. Nikolaizik WH, Preece MA, Warner JO. One year follow-up study of endocrine and lung function of asthmatic children on inhaled budesonide. Eur Respir J 1997; 10: 2596-2601. 
25. Prahl P, Jensen T, Bjerregaard-Andersen H. Adrenocortical function in children on high dose steroid aerosol therapy. Results of serum cortisol, ACTH stimulation test and 24 hour urinary free cortisol excretion. Allergy 1987; 42: 541-544.

26. Goldstein DE, König P. Effect of inhaled beclomethasone dipropionate on hypothalamic-pituitary-adrenal axis function in children with asthma. Pediatr 1983; 72: 60-64.

27. Yiallouros PK, Milner AD, Conway E, Honour JW. Adrenal function and high dose inhaled corticosteroids for asthma. Arch Dis Child 1997; 76: 405-410.

28. Jennings BH, Andersson KE, Johansson SA. Assessment of systemic effects of inhaled glucocorticoids: comparison of effects of inhaled budesonide and oral prednisolone on adrenal function and markers of bone turnover. Eur J Clin Pharmacol 1991; 40: 77-82.

29. Nikolaizik WH, Marchant JL, Preece MA, Warner JO. Endocrine and lung function in asthmatic children on inhaled corticosteroids. Am J Respir Crit Care Med 1994; 150: 624-628.

30. Birkebæk NH, Esberg G, Andersen K, Wolthers O, Hassager $\mathrm{C}$. Bone and collagen turnover during treatment with inhaled dry powder budesonide and beclomethasone dipropionate. Arch Dis Child 1995; 73: 524-527.

31. König P, Hillman L, Cervantes C, et al. Bone metabolism in children with asthma treated with inhaled beclomethasone dipropionate. J Pediatr 1993; 122: 219-226.

32. Martinati LC, Bertoldo F, Gasperi E, Micelli S, Boner A. Effect on cortical and trabecular bone mass of different anti-inflammatory treatments in preadolescent children with chronic asthma. Am J Respir Crit Care Med 1996; 153: 232-236.

33. Littlewood JM, Johnson AW, Edwards PA, Littlewood AK. Growth retardation in asthmatic children treated with inhaled beclomethasone dipropionate (Letter). Lancet 1988; i: 115-116.

34. Tinkleman DG, Reed CE, Nelson HS, Offord KP. Aerosol beclomethasone dipropionate compared with theophylline as primary treatment of chronic, mild to moderately severe asthma in children. Pediatrics 1993; 92: 64-77.

35. Wolthers OD, Pederson S. Short term growth during treatment with inhaled fluticasone propionate and beclomethasone dipropionate. Arch Dis Child 1993; 68: 673-676.

36. Crowley S, Hindmarsh PC, Matthews DR, Brook CGD. Growth and the growth hormone axis in prepubertal children with asthma. J Pediatr 1996; 126: 297-303.

37. Verberne AAPH, Frost C, Roorda RJ, et al. One year treatment with salmeterol compared with beclomethasone in children with asthma. Am J Respir Crit Care Med 1997; 156: 688-695.

38. Simons FE. A comparison of beclomethasone, salmeterol and placebo in children with asthma. N Engl J Med 1997; 337: 1690-1692.

39. Silverstein MD, Yunginger JW, Reed CE, O'Fallon WM. Attained adult height after childhood asthma: effect of glucocorticoid therapy. J Allergy Clin Immunol 1997; 99: 466-474. 